\title{
Comparative Analysis of Luteolin and Luteolin-7-O-Glucoside on anti-Atherogenesis in ApoE Knockout Mice with Hyperhomocysteinemia
}

\section{Abstract}

Luteolin is a naturally occurring flavone that reportedly has anti-inflammatory effect. Flavones in plants are usually present in the form of glucosides, although occasionally they are found as aglycones. The bioavailability of flavones may differ when consumed as either aglycones or glucosides. Nonetheless, numerous studies focused on the biological activity of flavonoid aglycones or that in vitro. These findings are supporting reason to compare the anti-atherogenic effect of aglycone and glucoside forms of flavones in vivo. Male ApoE knockout mice ( $\mathrm{n}=28$, 6-week-old) were divided randomly into 4 groups of 7 mice: negative control group, homocysteine control group, luteolin and luteolin7- $O$-glucoside groups with homocysteine. All animals were fed by a high-fat diet, modified by AIN-93, containing $0.5 \%$ of cholesterol and $45 \%$ of fat. Luteolin and luteolin-7-O-glycoside were given daily by gavage for 5 weeks $(50 \mathrm{mg} / \mathrm{kg} \mathrm{BW}$, respectively). To induce hyperhomocysteinemia, homocysteine was provided as a drinking water $(0.9 \mathrm{~g} / \mathrm{L})$. Administration of homocysteine did not affect body weight gain, feed intake and feed efficiency ratio among groups. Homocysteine feeding sharply increased serum concentrations of homocysteine and triglyceride as well as adhesion molecules including monocyte chemoattractant protein-1 and vascular cell adhesion molecule-1, which were attenuated by the administration of luteolin and luteolin-7-O-glucoside $(\mathrm{p}<0.05)$. Homocysteine administration produced development of atherosclerotic process by the induction of hepatic inducible nitric oxide synthase and cyclooxygenase-2 as well as aortic intercellular adhesion molecule expressions along with diminished expressions of antioxidative enzymes, such as hepatic glutathione reductase (GR), aortic GR and glutathione peroxidase $(\mathrm{p}<0.05)$. Administration of both flavones down-regulated expressions of inflammatory mediators and adhesion molecules as well as up-regulated expressions of antioxidative enzymes $(p<0.05)$. These data were in accordance with the histopathological observations which were analyzed by hematoxylin and eosin (H\&E) stain and immunohistochemistry. In a comparison of both agents, luteolin more potently attenuated inflammation and oxidative stress than luteolin-7- $O$-glucoside. These results exhibit that luteolin and luteolin-7-O-glycoside ameliorated atherogenic processes through the regulation of inflammation and oxidative stress in ApoE knockout mice with hyperhomocysteinemia. This work was supported by the National Research Foundation of Korea (NRF) grant funded by the Korea government of MOE (No 201704340001).

\section{Conflict of Interest}

There is no conflict of interest. 